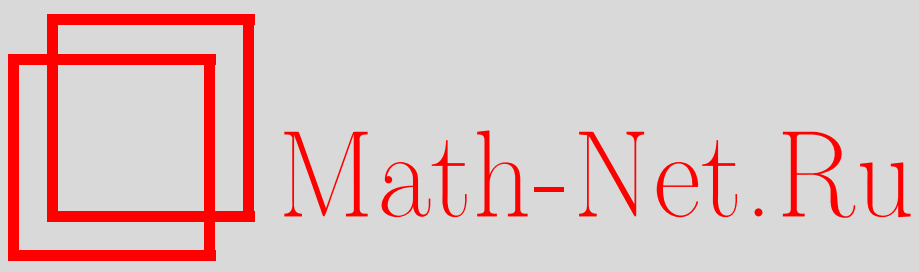

К. С. Сперанский, П. А. Терехин, О существовании фреймов в пространстве Харди, построенных на основе ядра Сеге, Изв. вузов. Матем., 2019, номер 2, 57-68

DOI: https://doi.org/10.26907/0021-3446-2019-2-57-68

Использование Общероссийского математического портала Math-Net.Ru подразумевает, что вы прочитали и согласны с пользовательским соглашением

http: //www. mathnet.ru/rus/agreement

Параметры загрузки:

IP : 52.23 .180 .231

26 апреля 2023 г., 17:38:14

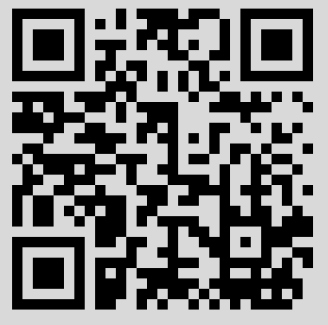


Известия вузов. Математика

2019, № 2, c. 57-68 https://kpfu.ru/science/nauchnye-izdaniya/ivrm/

e-mail: izvuz.matem@kpfu.ru

К.С. СПЕРАНСКИЙ, П.А. ТЕРЕХИН

\title{
О СУЩЕСТВОВАНИИ ФРЕЙМОВ В ПРОСТРАНСТВЕ ХАРДИ, ПОСТРОЕННЫХ НА ОСНОВЕ ЯДРА СЕГЕ
}

\begin{abstract}
Аннотация. Хорошо известно, что последовательность функций, состоящая из значений воспроизводящего ядра Сеге пространства Харди в единичном круге, не может быть фреймом Даффина-Шеффера. В данной работе показано, что при использовании более общей концепции фрейма вопрос о существовании такого обобщенного фрейма, построенного на основе ядра Сеге, решается положительно.
\end{abstract}

Ключевые слова: фреймы Даффина-Шеффера, банаховы фреймы, модельное пространство фрейма, воспроизводящее ядро гильбертова пространства, пространство Харди, ядро Сеге.

УДК: 517.518

DOI: $10.26907 / 0021-3446-2019-2-57-68$

\section{ВВЕДЕНИЕ}

Задача представления функций рядами по элементам заданной последовательности $e_{n}$, $n=1,2, \ldots$, заключается в нахождении для произвольной функции $f$ из некоторого класса $F$ последовательности коэффициентов $c_{n}, n=1,2, \ldots$, такой, что $f=\sum_{n=1}^{\infty} c_{n} e_{n}$. Вопросы представления функций рядами составляют обширную область теории функций и имеют многочисленные приложения. Наряду с классическими методами решения задачи представления активно развиваются современные методы представления на основе использования орторекурсивных разложений (Т.П. Лукашенко [1], В.В. Галатенко, Т.П. Лукашенко и В.Н. Садовничий [2]), жадных алгоритмов (В.Н. Темляков [3]), а также теории фреймов, предоставляющей общие и даже универсальные подходы к конструкции представления. Здесь следует упомянуть классическую работу Р. Даффина и А. Шеффера [4], в которой введено понятие фрейма гильбертова пространства, и теорию Файхтингера и К. Грохенига [5], [6], особенно работу К. Грохенига [7], в которой концепция фрейма впервые распространена на ситуацию банахова пространства. Понятия атомарного разложения и банахова фрейма по Грохенигу легли в основу других близких определений, например, фрейма Шаудера (П. Касазза, Д. Хан и Д. Ларсон [8]) и $X_{d}$-фрейма (П. Касазза, О. Кристенсен и Д. Стоева $[9])$.

Задача представления тесно связана, в частности, с задачей восстановления функции по заданной последовательности ее значений, что приводит к вопросу о фреймовых (представляющих) свойствах системы функций, состоящей из значений воспроизводящего ядра $K\left(\cdot, x_{n}\right), n=1,2, \ldots$, функционального гильбертова пространства. Ответ на данный вопрос

Поступила в редакцию 05.12.2017, после доработки 09.01.2018. Принята к публикации 22.03.2018

Благодарности. Работа выполнена при финансовой поддержке Российского фонда фундаментальных исследований (проект № 18-01-00414). 
существенно зависит от геометрии пространства. Например, для пространства Харди $H^{2}(\mathbb{D})$ хорошо известно (П. Дюрен и Шустер [10], гл. 6), что не существует фрейма ДаффинаШеффера в $H^{2}(\mathbb{D})$, построенного на основе ядра Сеге. А для собственного подпространства $K \subset H^{2}(\mathbb{D})$, порожденного функциями $K\left(\cdot, z_{n}\right), n=1,2, \ldots$, критерием последовательности Рисса (нормированного ядра) является условие Карлесона (конечно, при выполнении условия Бляшке), что также эквивалентно тому, что точки $\left\{z_{n}\right\}_{n=1}^{\infty}$ образуют интерполяционную последовательность. Эти и некоторые другие хорошо известные представляющие свойства ядра Сеге приводим в разделе 1.

Основной вопрос, рассматриваемый в настоящей работе, заключается в следующем:

существует ли фрейм пространства Харди, построенный на основе ядра Сеге?

Естественно, речь идет об использовании определения понятия фрейма, более общего, нежели фрейм Даффина-Шеффера. Даем положительный ответ на основной вопрос (теорема 3$)$, останавливаясь на понятии фрейма $\left\{e_{n}\right\}_{n=1}^{\infty}$ в банаховом пространстве $F$ относительно модельного пространства последовательностей $\mathcal{E}$ (определение 3 ).

\section{1. ПРЕДВАРИТЕЛЬНЫЕ СВЕДЕНИЯ}

Пусть $H$ - сепарабельное комплексное гильбертово пространство. Последовательность $\left\{e_{n}\right\}_{n=1}^{\infty}$ ненулевых элементов пространства $H$ называется фреймом Даффина-Шеффера, если существуют постоянные $0<A \leq B<\infty$ такие, что для всех $f \in H$ выполняются неравенства

$$
A\|f\|^{2} \leq \sum_{n=1}^{\infty}\left|\left(f, e_{n}\right)\right|^{2} \leq B\|f\|^{2} .
$$

Термин фрейм был предложен Р. Даффином и А. Шеффером [4]. Двусторонняя оценка (1) ранее рассматривалась в работе Бари [11] в качестве обобщения равенства Парсеваля. Построению фреймов в конкретных гильбертовых пространствах на основе систем функций специального вида (фреймы экспонент, фреймы сдвигов, фреймы всплесков) посвящены многочисленные исследования. В монографии О. Кристенсена [12] приведены классические и современные результаты, а также обширная библиография по данной тематике. Интерес к построению фреймов обусловлен, в частности, тем обстоятельством, что всякий фрейм Даффина-Шеффера $\left\{e_{n}\right\}_{n=1}^{\infty}$ является системой представления в $H$ относительно $\ell^{2}$, т. е. для любого $f \in H$ существует числовая последовательность $\left\{\xi_{n}\right\}_{n=1}^{\infty} \in \ell^{2}$, для которой справедливо представление

$$
f=\sum_{n=1}^{\infty} \xi_{n} e_{n} .
$$

В связи с задачей восстановления функции $f$ по дискретному (счетному) набору ее значений $\left\{f\left(x_{n}\right)\right\}_{n=1}^{\infty}$ особую роль играют вопросы построения фреймов на основе воспроизводящих ядер функциональных гильбертовых пространств. Напомним соответствующие определения.

Определение 1. Пусть $X$ - некоторое непустое множество. Гильбертово пространство $H$ называется функииональным (над $X$ ), если каждый элемент $f \in H$ является функцией $f: X \rightarrow \mathbb{C}$ и при этом для всех $x \in X$ линейный функционал $\delta_{x}(f)=f(x)$ на $H$, который называется оценочным функиионалом, корректно определен и непрерывен, т. е. найдется $C_{x}<\infty$ такое, что для всех $f \in H$ выполняется неравенство

$$
|f(x)| \leq C_{x}\|f\| .
$$


По теореме Рисса о представлении непрерывного линейного функционала в гильбертовом пространстве для каждого $x \in X$ существует и при том единственный элемент $K_{x} \in H$ такой, что

$$
\delta_{x}(f)=\left(f, K_{x}\right), \quad f \in H .
$$

Определение 2. Функция $K: X \times X \rightarrow \mathbb{C}$, определенная посредством равенства

$$
K(x, t)=\left(K_{t}, K_{x}\right), \quad x, t \in X,
$$

называется воспроизводящим ядром функционального гильбертова пространства $H$.

Пусть $x_{n}, n=1,2, \ldots$, - попарно различные точки множества $X$. Рассмотрим вопрос о фреймовых (представляющих) свойствах в $H$ нормированной последовательности

$$
e_{n}=\left\|K_{x_{n}}\right\|^{-1} K_{x_{n}}, \quad n=1,2, \ldots .
$$

Сразу заметим, что ответ на этот вопрос принципиальным образом зависит не только от выбора точек, но и от геометрии функционального гильбертова пространства. В данной работе ограничиваемся выбором в качестве $H$ пространства Харди. Также отметим, что известен широкий круг задач, связывающих в единое целое проблематику фреймов и воспроизводящих ядер. Здесь упомянем монографию Дж. Партингтона ([13], гл. 5) (в особенности §5.5, где указывается на взаимосвязь непрерывного всплескового преобразования с воспроизводящим ядром подходящего гильбертового пространства); статью [14], посвященную фреймам и ядрам в ситуации банахова пространства с внутренним произведением (частным случаем которого является скалярное произведение); статью [15], в которой решается обратная задача построения ядра по заданному фрейму; статью [16], где изучаются близкие вопросы плотности сэмплинга и интерполяции на основе ядер; а также статьи [17], [18], сочетающие в себе теоретические и прикладные вопросы взаимосвязи фреймов и воспроизводящих ядер. Конечно, приведенные отдельные ссылки не претендуют на полноту.

Теперь перейдем к постановке частного случая рассматриваемого вопроса, касающегося фреймовых (представляющих) свойств ядра Сеге пространства Харди.

Пространство Харди $H^{2}=H^{2}(\mathbb{D})$ состоит из всех аналитических в единичном круге $\mathbb{D}=(|z|<1)$ функций $f(z)$, для которых конечна норма

$$
\|f\|_{2}=\sup _{0<r<1}\left(\frac{1}{2 \pi} \int_{0}^{2 \pi}\left|f\left(r e^{i t}\right)\right|^{2} d t\right)^{1 / 2}<\infty .
$$

Пространство $H^{2}$ является функциональным гильбертовым пространством с воспроизводящим ядром

$$
K(z, \zeta)=K_{\zeta}(z)=\frac{1}{1-\bar{\zeta} z}, \quad z, \zeta \in \mathbb{D},
$$

которое называется ядром Сеге (например, [19]). Для попарно различных точек $\left\{z_{n}\right\}_{n=1}^{\infty} \subset \mathbb{D}$ полагаем

$$
e_{n}=\left(1-\left|z_{n}\right|^{2}\right)^{1 / 2} K_{z_{n}}, \quad n=1,2, \ldots,
$$

- нормированная в $H^{2}$ (т.е. $\left\|e_{n}\right\|_{2}=1$ ) последовательность дискретизаций ядра Сеге $K\left(\cdot, z_{n}\right)$. Следующие свойства последовательности $\left\{e_{n}\right\}_{n=1}^{\infty}$ хорошо известны (например, [13], гл. 2).

Предложение 1. Последовательность $\left\{e_{n}\right\}_{n=1}^{\infty}$ является полной в $H^{2}$ в том и только том случае, если

$$
\sum_{n=1}^{\infty}\left(1-\left|z_{n}\right|\right)=\infty
$$


Предложение 2. Последовательность $\left\{e_{n}\right\}_{n=1}^{\infty}$ является минимальной в $H^{2}$ тогда и только тогда, когда

$$
\sum_{n=1}^{\infty}\left(1-\left|z_{n}\right|\right)<\infty
$$

Условие (В) называется условием Бляшке. Поскольку условие (А) является отрицанием условия Бляшке, то последовательность $\left\{e_{n}\right\}_{n=1}^{\infty}$ не может быть одновременно полной и минимальной, в частности, базисом пространства $H^{2}$.

Напомним, что $\left\{e_{n}\right\}_{n=1}^{\infty} \subset H$ называется последовательностью Рисса, если существуют постоянные $0<A \leq B<\infty$ такие, что для любой числовой последовательности $\left\{c_{n}\right\}_{n=1}^{\infty} \in$ $\ell^{2}$ выполняются неравенства

$$
A \sum_{n=1}^{\infty}\left|c_{n}\right|^{2} \leq\left\|\sum_{n=1}^{\infty} c_{n} e_{n}\right\|_{H}^{2} \leq B \sum_{n=1}^{\infty}\left|c_{n}\right|^{2} .
$$

Предложение 3. Пусть точки $\left\{z_{n}\right\}_{n=1}^{\infty}$ удовлетворяют условию (В). Тогда следующие утверждения эквивалентны:

(i) $\left\{e_{n}\right\}_{n=1}^{\infty}$ является последовательностъю Рисса;

(ii) $\left\{e_{n}\right\}_{n=1}^{\infty}$ является равномерно минимальной последовательностью;

(iii) существует постоянная $\delta>0$ такая, что

$$
\prod_{m \neq n}\left|\frac{z_{m}-z_{n}}{1-\bar{z}_{n} z_{m}}\right| \geq \delta>0, \quad n=1,2, \ldots .
$$

Условие $(\mathrm{C})$ называется условием Карлесона и эквивалентно тому, что точки $\left\{z_{n}\right\}_{n=1}^{\infty}$ образуют интерполяиионную последовательность: для всех $\left\{w_{n}\right\}_{n=1}^{\infty} \in \ell^{\infty}$ существует функция $f \in H^{\infty}(\mathbb{D})$, для которой $f\left(z_{n}\right)=w_{n}, n=1,2, \ldots$ Условие Нъюмана заключается в существовании постоянной $B<\infty$ такой, что для всех $f \in H^{2}$ выполнено неравенство

$$
\sum_{n=1}^{\infty}\left(1-\left|z_{n}\right|^{2}\right)\left|f\left(z_{n}\right)\right|^{2} \leq B\|f\|_{2}^{2} .
$$

Это условие означает, что $\left\{e_{n}\right\}_{n=1}^{\infty}$ является последовательностъю Бесселя. На основании предложения 3 из условий $(B)$ и $(C)$ вытекает условие $(N)$. Кроме того, имеет место

Предложение 4. Каждое множество точек $\left\{z_{n}\right\}_{n=1}^{\infty}$, удовлетворяющее условию $(\mathrm{N})$, является конечным объединением интерполячионных последовательностей.

Это предложение отражает следующий общий факт.

Каждая последовательность Бесселя $\left\{h_{n}\right\}_{n=1}^{\infty}, \inf _{n=1,2, \ldots}\left\|h_{n}\right\|>0$, является конечным объединением последовательностей Рисса.

Это замечательное утверждение было высказано в качестве гипотезы Файхтингером и эквивалентно гипотезе Кадисона-Зингера, доказаной в 2013 г. А. Маркусом, Д. Шпильманом и Н. Шриваставой [20].

Из очевидной импликации $(\mathrm{N}) \Rightarrow(\mathrm{B})$ вытекает

Предложение 5. Всякая последовательность Бесселя $\left\{e_{n}\right\}_{n=1}^{\infty}$ заведомо не полна в $H^{2}$, в частности, не существует точек $\left\{z_{n}\right\}_{n=1}^{\infty}$, для которых $\left\{e_{n}\right\}_{n=1}^{\infty}$ образует фрейм ДафбинаШеффера.

Таким образом, условие $\ell^{2}$-стабильности (1) из определения фрейма Даффина-Шеффера не выполнимо для последовательности $\left\{e_{n}\right\}_{n=1}^{\infty}$. Неформально говоря, $\ell^{2}$-норма слишком 
"мала" (и пространство $\ell^{2}$ слишком "велико"), чтобы обеспечить оценку снизу в (1) при наличии оценки сверху. Однако далее будет показано, что при подходящем обобщении понятия фрейма искомый обобщенный фрейм вида $\left\{e_{n}\right\}_{n=1}^{\infty}$ может быть построен. Раздел 2 содержит обсуждение рассматриваемого определения фрейма и, наконец, в разделе 3 приводим искомую конструкцию, которая автоматически гарантирует возможность представления вида (2) для произвольной функции $f \in H^{2}$.

Замечание 1. Ситуация, при которой свойство полноты системы функций из некоторого класса входит в прямое противоречие с другими естественными свойствами такими, как минимальность или бесселевость, происходит во многих классических случаях. Например, для систем степеней $\left\{x^{p_{k}}\right\}_{k=1}^{\infty}$ минимальность в $L^{2}(a, b)$ равносильна отрицанию условия Мюнца, эквивалентного полноте (например, [21], гл. $1, \S 13)$. Для систем сдвигов $\left\{\varphi\left(x-\lambda_{k}\right)\right\}_{k \in \mathbb{Z}}$ бесселевость в $L^{2}(\mathbb{R})$ имеет следствием конечную верхнюю плотность Берлинга точек $\left\{\lambda_{k}\right\}_{k \in \mathbb{Z}}$, в то время как существование нижней границы фрейма Даффина-Шеффера (из которого вытекает полнота) имплицирует бесконечную верхнюю плотность Берлинга и поэтому в $L^{2}(\mathbb{R})$ не существует фреймов Даффина-Шеффера из сдвигов одной функции ([12], гл. 9, $\S 6)$. Для систем дискретизированных воспроизводящих ядер гильбертовых пространств подобные тонкие результаты получены в статье [16].

Замечание 2. Анти-условие Бляшке (А), т. е. отрицание (В), гарантирует переполненность последовательности $\left\{e_{n}\right\}_{n=1}^{\infty}$ : для каждого $n_{0} \in \mathbb{N}$ подпоследовательность $\left\{e_{n}\right\}_{n=n_{0}}^{\infty}$ также будет полной в $H^{2}$. Следовательно, для любой функции $f \in H^{2}$ найдется $\left\{n_{k}\right\}_{k=1}^{\infty} \subset \mathbb{N}$ такое, что справедливо представление (полагаем $n_{0}=1$ )

$$
f=\sum_{k=1}^{\infty} \sum_{n=n_{k-1}}^{n_{k}-1} \xi_{n} e_{n} .
$$

Такое представление еще не означает, что $\left\{e_{n}\right\}_{n=1}^{\infty}$ является системой представления в $H^{2}$, поскольку номера $n_{k}$, вообще говоря, зависят от $f$. Кроме того, теорема Тотика [22] утверждает, что условие (A) необходимо и достаточно для решения задачи восстановления, а именно, в предположении (А) существует семейство алгебраических полиномов $p_{n, k}$, где $n=1,2, \ldots$ и $k=1, \ldots, n$, таких, что для любой функции $f \in H^{2}$ справедливо представление

$$
f=\lim _{n \rightarrow \infty} \sum_{k=1}^{n} f\left(z_{k}\right) p_{n, k} .
$$

Ясно, что такое аппроксимационное представление также, вообще говоря, не гарантирует представления в виде ряда (2).

\section{2. ФРЕЙМЫ И ИХ МОДЕЛЬНЫЕ ПРОСТРАНСТВА}

Всюду далее $\mathcal{E}$ - банахово пространство последовательностей с естественным базисом $\varepsilon_{i}=\left\{\delta_{i j}\right\}_{j=1}^{\infty}, i=1,2, \ldots(\delta-$ символ Кронекера $)$. Такое пространство $\mathcal{E}$ будем называть моделъным. Каждый непрерывный линейный функционал $l$ на $\mathcal{E}$ однозначно определяется своими значениями $\left\{l\left(\varepsilon_{n}\right)\right\}_{n=1}^{\infty}$ на элементах естественного базиса. Поэтому сопряженное пространство $\mathcal{E}^{*}$ изометрически изоморфно некоторому пространству последовательностей и будет далее отождествляться с ним.

Пусть $F$ - сепарабельное комплексное банахово пространство и $G=F^{*}$ сопряженное к нему. Как обычно, $(f, g)$ означает значение функционала $g \in G$ на элементе $f \in F$. 
Определение 3. Последовательность $\left\{e_{n}\right\}_{n=1}^{\infty}$ ненулевых элементов банахова пространства $F$ называется фреймом в $F$ относительно $\mathcal{E}$, если существуют постоянные $0<A \leq B<\infty$ такие, что для всех $g \in G$ выполняются неравенства

$$
A\|g\|_{G} \leq\left\|\left\{\left(e_{n}, g\right)\right\}_{n=1}^{\infty}\right\|_{\mathcal{E}^{*}} \leq B\|g\|_{G} .
$$

Данное определение является двойственным по отношению к известным определениям атомарного разложения [7], а также концепции фрейма Шаудера [8], понятию $X_{d}$-фрейма [9] и было предложено в статье [23]. Как показано в [23], определение 3 дает универсальный способ решения задачи представления функций рядами, а именно, имеет место

Теорема 1. Последовательность $\left\{e_{n}\right\}_{n=1}^{\infty}$ является фреймом в $F$ относительно $\mathcal{E}$ тогда и только тогда, когда выполняются следующие два условия:

(i) $\left\{e_{n}\right\}_{n=1}^{\infty}$ является системой сходимости в $F$ относительно $\mathcal{E}$, m. е. для всех $\xi=$ $\left\{\xi_{n}\right\}_{n=1}^{\infty} \in \mathcal{E}$ ряд $\sum_{n=1}^{\infty} \xi_{n} e_{n}$ сходится в $F$

(ii) $\left\{e_{n}\right\}_{n=1}^{\infty}$ является системой представления в $F$ относительно $\mathcal{E}$, m. е. для всех $f \in F$ существует последовательность $\xi=\left\{\xi_{n}\right\}_{n=1}^{\infty} \in \mathcal{E}$ такал, что $f=\sum_{n=1}^{\infty} \xi_{n} e_{n}$.

Замечание 3. В настоящее время насчитывается более десятка неэквивалентных между собой определений понятия фрейма в ситуации банахова пространства. Соотношение с ними выбранного определения 3 обсуждается в работах [24] и [25]. Следует заметить, что если $F=$ $G=H$ - гильбертово пространство, то фреймовые неравенства (3) могут быть записаны в виде

$$
A\|f\| \leq\left\|\left\{\left(f, e_{n}\right)\right\}_{n=1}^{\infty}\right\|_{\mathcal{E}^{*}} \leq B\|f\|
$$

и поэтому совпадают в этом частном случае с фреймовыми неравенствами из определений работ [7] - [9]. Если к тому же $\mathcal{E}=\mathcal{E}^{*}=\ell^{2}$, то получаем фреймовые неравенства ДаффинаШеффера (1) с точностью до извлечения квадратного корня из постоянных.

Замечание 4. Выбор модельного пространства $\mathcal{E}$ фрейма $\left\{e_{n}\right\}_{n=1}^{\infty}$ оказывает влияние на тип сходимости представляющего $f$ ряда. Например, если естественный базис пространства $\mathcal{E}$ является безусловным, то ряд в правой части представления (2) (с коэффициентами из $\mathcal{E})$ безусловно сходится к $f$. Если же пространство $\mathcal{E}$ является $\ell^{1}$-суммой конечномерных координатных пространств $\mathcal{E}_{k}, \operatorname{dim} \mathcal{E}_{k}=n_{k}$,

$$
\mathcal{E}=\left(\bigoplus_{k=1}^{\infty} \mathcal{E}_{k}\right)_{\ell^{1}},
$$

что соответствует разбиению последовательности $\left\{e_{n}\right\}_{n=1}^{\infty}$ на блоки

$$
e_{n}=e_{k, j}, \quad j=0, \ldots, n_{k}-1, \quad k=1,2, \ldots,
$$

то ряд в правой части представления (2) абсолютно сходится к $f$ по блокам

$$
\sum_{k=1}^{\infty}\left\|\sum_{j=0}^{n_{k}-1} \xi_{k, j} e_{k, j}\right\|_{F}<\infty .
$$

В этом случае для подпространств $F_{k}=\operatorname{span}\left\{e_{k, j}\right\}_{j=0}^{n_{k}-1} \subset F$ имеем

$$
F=\left(\bigoplus_{k=1}^{\infty} F_{k}\right)_{\ell^{1}} .
$$


В следующем разделе будет рассмотрена именно такая ситуация и поэтому после построения фрейма $\left\{e_{n}\right\}_{n=1}^{\infty}$ на основе ядра Сеге будут справедливы подобные представления. Также будем использовать двойственность $\ell^{1}$ - и $\ell^{\infty}$-сумм, которая заключается в том, что

$$
\mathcal{E}^{*}=\left(\bigoplus_{k=1}^{\infty} \mathcal{E}_{k}^{*}\right)_{\ell^{\infty}}
$$

\section{3. ПоСТРОеНИЕ ФРЕЙМА НА ОСНОВЕ ЯДРА СЕГЕ}

Дискретное преобразование Фурье - это унитарный оператор в $n$-мерном унитарном пространстве $\ell_{n}^{2}$, определяемый равенством

$$
\widehat{\xi}_{k}=\frac{1}{\sqrt{n}} \sum_{j=0}^{n-1} \xi_{j} e^{-2 \pi i j k / n}, \quad k=0, \ldots, n-1 .
$$

Пусть $0<r<1$. Положим в соответствии с принятыми ранее обозначениями

$$
e_{j}=\left(1-r^{2}\right)^{1 / 2} K_{\zeta_{j}}, \quad \zeta_{j}=r e^{2 \pi i j / n}, \quad j=0, \ldots, n-1 .
$$

Лемма. Для всех $n \in \mathbb{N} u r \in(0,1)$ выполняются неравенства

$$
\frac{r^{2 n} n\left(1-r^{2}\right)}{1-r^{2 n}} \sum_{j=0}^{n-1}\left|\xi_{j}\right|^{2} \leq\left\|\sum_{j=0}^{n-1} \xi_{j} e_{j}\right\|_{2}^{2} \leq \frac{n\left(1-r^{2}\right)}{1-r^{2 n}} \sum_{j=0}^{n-1}\left|\xi_{j}\right|^{2} .
$$

Доказательство. По определению ядра Сеге

$$
\sum_{j=0}^{n-1} \xi_{j} e_{j}(z)=\sum_{j=0}^{n-1} \frac{\xi_{j}\left(1-r^{2}\right)^{1 / 2}}{1-r e^{-2 \pi i j / n} z}=\left(1-r^{2}\right)^{1 / 2} \sum_{k=0}^{\infty} r^{k}\left(\sum_{j=0}^{n-1} \xi_{j} e^{-2 \pi i j k / n}\right) z^{k},
$$

откуда

$$
\left\|\sum_{j=0}^{n-1} \xi_{j} e_{j}\right\|_{2}^{2}=\left(1-r^{2}\right) \sum_{k=0}^{\infty} r^{2 k}\left|\sum_{j=0}^{n-1} \xi_{j} e^{-2 \pi i j k / n}\right|^{2} .
$$

Учитывая периодичность экспонент по модулю $n$, т. е. $e^{-2 \pi i j(l n+k) / n}=e^{-2 \pi i j k / n}$, находим

$$
\left\|\sum_{j=0}^{n-1} \xi_{j} e_{j}\right\|_{2}^{2}=\left(1-r^{2}\right) \sum_{l=0}^{\infty} r^{2 l n} \sum_{k=0}^{n-1} r^{2 k}\left|\sum_{j=0}^{n-1} \xi_{j} e^{-2 \pi i j k / n}\right|^{2}=\frac{n\left(1-r^{2}\right)}{1-r^{2 n}} \sum_{k=0}^{n-1} r^{2 k}\left|\widehat{\xi}_{k}\right|^{2} .
$$

Используя тривиальные оценки $r^{2 n}<r^{2 k}<1$ и унитарность дискретного преобразования Фурье, получаем искомые неравенства.

Рассмотрим точки $\left\{z_{n}\right\}_{n=1}^{\infty}$ специального вида

$$
z_{n}=z_{k, j}=r_{k} e^{2 \pi i j / n_{k}}, \quad j=0, \ldots, n_{k}-1, \quad k=1,2, \ldots,
$$

где

$$
n_{1}<\cdots<n_{k}<\cdots, \quad 0<r_{1}<\cdots<r_{k}<\cdots, \quad \lim _{k \rightarrow \infty} r_{k}=1,
$$

и потребуем, чтобы "номера" $n_{k}$ и "радиусы" $r_{k}$ удовлетворяли следующему условию согласования: существуют постоянные $0<a \leq b<\infty$ такие, что

$$
a / n_{k} \leq 1-r_{k} \leq b / n_{k}, \quad k=1,2, \ldots .
$$

Далее будем использовать обозначение

$$
\Omega:=\left\{(k, j): j=0, \ldots, n_{k}-1, k=1,2, \ldots\right\}
$$


для множества пар $(k, j)$, индексирующих точки $z_{n}=z_{k, j}$, и соответствующие им значения нормированного ядра Сеге $e_{n}=e_{k, j}$.

Теорема 2. Пусть точки $\left\{z_{n}\right\}_{n=1}^{\infty}$ имеют вид (4) и удовлетворяют условию (5). Тогда следующие утверждения эквивалентны:

(i) для коэффичиентов $\xi_{n}=\xi_{k, j}$ ряда $\sum_{n=1}^{\infty} \xi_{n} e_{n}$ выполняется условие

$$
\sum_{k=1}^{\infty}\left(\sum_{j=0}^{n_{k}-1}\left|\xi_{k, j}\right|^{2}\right)^{1 / 2}<\infty
$$

(ii) ряд $\sum_{n=1}^{\infty} \xi_{n} e_{n}$ абсолютно сходится по блокам в $H^{2}$, т.е.

$$
\sum_{k=1}^{\infty}\left\|\sum_{j=0}^{n_{k}-1} \xi_{k, j} e_{k, j}\right\|_{2}<\infty .
$$

При этом ряд $\sum_{n=1}^{\infty} \xi_{n} e_{n}$ безусловно сходится в $H^{2}$.

Доказательство. На основании леммы для всех $k=1,2, \ldots$ имеем

$$
A_{k} \sum_{j=0}^{n_{k}-1}\left|\xi_{k, j}\right|^{2} \leq\left\|\sum_{j=0}^{n_{k}-1} \xi_{k, j} e_{k, j}\right\|_{2}^{2} \leq B_{k} \sum_{j=0}^{n_{k}-1}\left|\xi_{k, j}\right|^{2}
$$

где

$$
A_{k}=\frac{r_{k}^{2 n_{k}} n_{k}\left(1-r_{k}^{2}\right)}{1-r_{k}^{2 n_{k}}}, \quad B_{k}=\frac{n_{k}\left(1-r_{k}^{2}\right)}{1-r_{k}^{2 n_{k}}} .
$$

В силу условия согласования (5), с одной стороны,

$$
r_{k}^{2 n_{k}} \geq\left(1-b / n_{k}\right)^{2 n_{k}} \rightarrow e^{-2 b}>0, \quad k \rightarrow \infty,
$$

откуда $r_{k}^{2 n_{k}} \geq \alpha>0, k=1,2, \ldots$. С другой стороны,

$$
r_{k}^{2 n_{k}} \leq\left(1-a / n_{k}\right)^{2 n_{k}} \rightarrow e^{-2 a}<1, \quad k \rightarrow \infty,
$$

откуда $r_{k}^{2 n_{k}} \leq \beta<1, k=1,2, \ldots$. Также ввиду (5) имеем $a \leq n_{k}\left(1-r_{k}^{2}\right) \leq 2 b, k=1,2, \ldots$. Следовательно, для всех $k$

$$
0<\frac{\alpha a}{1-\alpha} \leq A_{k} \leq B_{k} \leq \frac{2 b}{1-\beta}<\infty .
$$

Отсюда

$$
\left(\frac{\alpha a}{1-\alpha}\right)^{1 / 2} \sum_{k=1}^{\infty}\left(\sum_{j=0}^{n_{k}-1}\left|\xi_{k, j}\right|^{2}\right)^{1 / 2} \leq \sum_{k=1}^{\infty}\left\|\sum_{j=0}^{n_{k}-1} \xi_{k, j} e_{k, j}\right\|_{2} \leq\left(\frac{2 b}{1-\beta}\right)^{1 / 2} \sum_{k=1}^{\infty}\left(\sum_{j=0}^{n_{k}-1}\left|\xi_{k, j}\right|^{2}\right)^{1 / 2} .
$$

Эквивалентность (i) $\Leftrightarrow$ (ii) установлена. Осталось показать, что из абсолютной сходимости по блокам следует безусловная сходимость ряда $\sum_{n=1}^{\infty} \xi_{n} e_{n}$. 
Предположим, что ряд $\sum_{n=1}^{\infty} \xi_{n} e_{n}$ абсолютно сходится по блокам в $H^{2}$. Возьмем $\varepsilon>0$ и, используя доказанную эквивалентность утверждений (i) и (ii), выберем $k_{0}$ так, чтобы

$$
\sum_{k=k_{0}}^{\infty}\left(\sum_{j=0}^{n_{k}-1}\left|\xi_{k, j}\right|^{2}\right)^{1 / 2}<\varepsilon
$$

Обозначим через $I_{0}$ множество всех индексов $(k, j) \in \Omega$, для которых $k<k_{0}$. Для произвольного конечного множества $I$ индексов $(k, j) \in \Omega$ такого, что $I \supset I_{0}$, имеем

$$
\left\|\sum_{(k, j) \in \Omega \backslash I} \xi_{k, j} e_{k, j}\right\|_{2} \leq \sum_{k=k_{0}}^{\infty}\left\|\sum_{\{j:(k, j) \in \Omega \backslash I\}} \xi_{k, j} e_{k, j}\right\|_{2}<\left(\frac{2 b}{1-\beta}\right)^{1 / 2} \varepsilon .
$$

Это означает, что семейство $\left\{\xi_{k, j} e_{k, j}\right\}_{(k, j) \in \Omega}$ является суммируемым, что равносильно его безусловной сходимости ([26], гл. 3, с. 120).

Доказанная теорема 2 побуждает к вполне оправданному выбору в качестве ассоциированного с системой $\left\{e_{n}\right\}_{n=1}^{\infty}$ модельного пространства

$$
\mathcal{E}=\left(\bigoplus_{k=1}^{\infty} \ell_{n_{k}}^{2}\right)_{\ell^{1}}
$$

Теорема 3. Пусть точки $\left\{z_{n}\right\}_{n=1}^{\infty}$ имеют вид (4) и удовлетворяют условию (5). Тогда последовательность $\left\{e_{n}\right\}_{n=1}^{\infty}$ является фреймом в $H^{2}$ относительно $\mathcal{E}$.

Доказательство. По теореме 2 корректно определен и ограничен оператор синтеза $S: \mathcal{E} \rightarrow H^{2}$, заданный равенством

$$
S \xi=\sum_{n=1}^{\infty} \xi_{n} e_{n}
$$

Действительно, полагая $B=\left(\frac{2 b}{1-\beta}\right)^{1 / 2}$, в обозначениях доказательства теоремы 2 имеем

$$
\|S \xi\|_{2} \leq \sum_{k=1}^{\infty}\left\|\sum_{j=0}^{n_{k}-1} \xi_{k, j} e_{k, j}\right\|_{2} \leq B\|\xi\|_{\mathcal{E}}
$$

Оператор анализа $R: H^{2} \rightarrow \mathcal{E}^{*}$, определяемый равенством

$$
R g=\left\{\left(e_{n}, g\right)\right\}_{n=1}^{\infty},
$$

является сопряженным к оператору синтеза, поскольку

$$
(S \xi, g)=\sum_{n=1}^{\infty} \xi_{n}\left(e_{n}, g\right)=(\xi, R g) .
$$

Следовательно, $\|R g\|_{\mathcal{E}^{*}} \leq B\|g\|_{2}$, что означает справедливость оценки сверху из фреймовых неравенств (3)

$$
\left\|\left\{\left(e_{n}, g\right)\right\}_{n=1}^{\infty}\right\|_{\mathcal{E}^{*}} \leq B\|g\|_{2} .
$$

Таким образом, для доказательства теоремы требуется получить оценку снизу в (3), что с учетом двойственности (см. замечание 4)

$$
\mathcal{E}^{*}=\left(\bigoplus_{k=1}^{\infty} \ell_{n_{k}}^{2}\right)_{\ell^{\infty}}
$$


и определения функций $e_{n}, n=1,2, \ldots$, записывается в виде

$$
\left\|\left\{\left(e_{n}, g\right)\right\}_{n=1}^{\infty}\right\|_{\mathcal{E}^{*}}=\sup _{k=1,2, \ldots}\left(\left(1-r_{k}^{2}\right) \sum_{j=0}^{n_{k}-1}\left|g\left(r_{k} e^{2 \pi i j / n_{k}}\right)\right|^{2}\right)^{1 / 2} \geq A\left\|_{g}\right\|_{2}
$$

Пусть сначала $g \in A(\mathbb{D})$, т. е. функция $g(z)$ аналитична в $\mathbb{D}$ и непрерывна в $\overline{\mathbb{D}}=(|z| \leq 1)$. В этом случае семейство функций $G_{r}(t)=\left|g\left(r e^{i t}\right)\right|^{2}, 0<r \leq 1$, равностепенно непрерывно и $G_{r}(t) \rightarrow G_{1}(t)$ при $r \rightarrow 1$ равномерно по $t$. Поэтому интегральные суммы Римана этих функций

$$
S\left(G_{r}, \varkappa_{n}\right)=\frac{2 \pi}{n} \sum_{j=0}^{n-1} G_{r}\left(\frac{2 \pi j}{n}\right)
$$

соответствующие разбиениям $\varkappa_{n}=\{2 \pi j / n\}_{j=0}^{n}$ отрезка $[0,2 \pi]$, сходятся к $\int_{0}^{2 \pi} G_{1}(t) d t=$ $2 \pi\|g\|_{2}^{2}$ при $r \rightarrow 1$ и $n \rightarrow \infty$. Действительно, имеем

$$
\left|S\left(G_{r}, \varkappa_{n}\right)-\int_{0}^{2 \pi} G_{r}(t) d t\right| \leq \sum_{j=0}^{n-1} \int_{2 \pi j / n}^{\frac{2 \pi(j+1)}{n}}\left|G_{r}(2 \pi j / n)-G_{r}(t)\right| d t \leq 2 \pi \omega\left(\left\{G_{r}\right\}_{0<r \leq 1}, 2 \pi / n\right),
$$

где модуль непрерывности семейства функций $G_{r}(t)$

$$
\omega\left(\left\{G_{r}\right\}_{0<r \leq 1}, \delta\right)=\sup _{0<r \leq 1} \sup _{|s-t|<\delta}\left|G_{r}(s)-G_{r}(t)\right|
$$

мажорируется модулем непрерывности функции $g(z)$

$$
\omega(g, \delta)=\sup _{|\zeta-z|<\delta}|g(\zeta)-g(z)|
$$

так как $|\zeta-z|=\left|r e^{i s}-r e^{i t}\right|=2 r \sin \frac{|s-t|}{2} \leq|s-t|$. Кроме того, имеем

$$
\left|\int_{0}^{2 \pi} G_{r}(t) d t-\int_{0}^{2 \pi} G_{1}(t) d t\right| \leq\left.\int_{0}^{2 \pi}|| g\left(r e^{i t}\right)\right|^{2}-\left|g\left(e^{i t}\right)\right|^{2} \mid d t \leq 2\|g\|_{A(\mathbb{D})} \cdot 2 \pi \omega(g, 1-r) .
$$

В итоге, как и утверждалось, при $r \rightarrow 1$ и $n \rightarrow \infty$

$$
\left|S\left(G_{r}, \varkappa_{n}\right)-\int_{0}^{2 \pi} G_{1}(t) d t\right| \leq C_{1} \omega\left(g, \frac{2 \pi}{n}\right)+C_{2} \omega(g, 1-r) \rightarrow 0 .
$$

В частности, имеет место предельное соотношение

$$
\lim _{k \rightarrow \infty}\left(\frac{1}{n_{k}} \sum_{j=0}^{n_{k}-1}\left|g\left(r_{k} e^{2 \pi i j / n_{k}}\right)\right|^{2}\right)^{1 / 2}=\|g\|_{2} .
$$

Поскольку $1 / n_{k} \leq\left(1-r_{k}^{2}\right) / a$ и $\lim \leq \sup$, то из последнего соотношения вытекает (7) с постоянной $A=\sqrt{a}>0$ для функции $g \in A(\mathbb{D})$. Пусть теперь функция $g \in H^{2}$ произвольна. Выберем $g_{\varepsilon} \in A(\mathbb{D})$ так, чтобы $\left\|g-g_{\varepsilon}\right\|_{2}<\varepsilon$. Тогда в силу $(6)$ имеем $\left\|R\left(g-g_{\varepsilon}\right)\right\| \mathcal{E}^{*}<B \varepsilon$ и поэтому

$$
\|R g\|_{\mathcal{E}^{*}} \geq\left\|R g_{\varepsilon}\right\|_{\mathcal{E}^{*}}-\left\|R\left(g-g_{\varepsilon}\right)\right\|_{\mathcal{E}^{*}} \geq A\left\|g_{\varepsilon}\right\|_{2}-B \varepsilon \geq A\|g\|_{2}-(A+B) \varepsilon .
$$

Так как $\varepsilon>0$ произвольно, то получаем (7) для всех $g \in H^{2}$. 
Следствие. Пусть точки $\left\{z_{n}\right\}_{n=1}^{\infty}$ имеют вид (4) и удовлетворяют условию (5). Тогда для любой функции $f \in H^{2}$ существует последовательность $\left\{\xi_{n}\right\}_{n=1}^{\infty}=\left\{\xi_{k, j}\right\}_{(k, j) \in \Omega}$ такая, что справедливо представление

$$
f=\sum_{n=1}^{\infty} \xi_{n}\left(1-\left|z_{n}\right|^{2}\right)^{1 / 2} K_{z_{n}}, \quad \sum_{k=1}^{\infty}\left(\sum_{j=0}^{n_{k}-1}\left|\xi_{k, j}\right|^{2}\right)^{1 / 2}<\infty .
$$

Замечание 5. Следует отметить, что коэффициенты данного представления $\xi_{n}=\xi_{n}(f)$, $n=1,2, \ldots$, нелинейно зависят от $f$. В частности, для каждого $z \in \mathbb{D}$ имеем

$$
K_{z}=\sum_{n=1}^{\infty} \xi_{n}(z)\left(1-\left|z_{n}\right|^{2}\right)^{1 / 2} K_{z_{n}}
$$

отсюда

$$
f(z)=\sum_{n=1}^{\infty}\left(1-\left|z_{n}\right|^{2}\right)^{1 / 2} f\left(z_{n}\right) \overline{\xi_{n}(z)}
$$

- формула восстановления в виде ряда, дающая при фиксированном z вычислительные преимущества по сравнению с аппроксимационной формулой восстановления Тотика (см. замечание 2).

\section{ЛИТЕРАТУРА}

[1] Лукашенко Т.П. О свойствах орторекурсивных разложений по неортогональным системам, Вестн. Московск. ун-та. Сер. 1. Матем. Механ. 1, 6-10 (2001).

[2] Галатенко В.В., Лукашенко Т.П., Садовничий В.А. О свойствах орторекурсивных разложений по подпространствам, Тр. МИАН 284, 138-141 (2014).

[3] Temlyakon V.N. Greedy approximation, Acta Numer. 17, 235-409 (2008).

[4] Duffin R.J., Schaeffer A.C. A class of nonharmonic Fourier series, Trans. Amer. Math. Soc. 72, 341-366 (1952).

[5] Feichtinger H.G., Gröchenig K. Banach spaces related to integrable group representations and their atomic decomposition. I, J. Funct. Anal. 86, 307-340 (1989).

[6] Feichtinger H.G., Gröchenig K. Banach spaces related to integrable group representations and their atomic decomposition. II, Monatsh. Math. 108, 129-148 (1989).

[7] Gröchenig K. Describing functions: atomic decompositions versus frames, Monatsh. Math. 112 (1), 1-41 (1991).

[8] Casazza P.G., Han D., Larson D.R. Frames for Banach spaces, Contemp. Math. 247, 149-182 (1999).

[9] Casazza P.G., Christensen O., Stoeva D. Frame expansion in separable Banach spaces, J. Math. Anal. Appl. 307 (2), 710-723 (2005).

[10] Duren P., Schuster A. Bergman spaces (AMS, Providence, RI, 2004).

[11] Бари Н.К. Биортогональные системы и базисы гилъбертова пространства, Матем. IV, Учен. зап. МГУ 148, 69-107 (1951).

[12] Christensen O. An introduction to frames and Riesz bases (2nd rev. ed., Appl. Numer. Harmon. Anal., Birkhäuser/Springer, New York, 2016).

[13] Partington J.R. Interpolation, indentification, and sampling (Clarendon Press, Oxford, 1997).

[14] Zhang H., Zhang J. Frames, Riesz bases, and sampling expansions in Banach spaces via semi-inner products, Appl. Comput. Harmon. Anal. 31, 1-25 (2011).

[15] Song M.-S., Jorgensen P.E.T. Reproducing kernel Hilbert space vs. frame estimates, Math. 3, 615-625 (2015).

[16] Führ H., Gröchenig K., Haimi A., Klotz A., Romero J.L. Density of sampling and interpolation in reproducing kernel Hilbert spaces, J. London Math. Soc. 96 (2), (2017).

[17] Gao J., Harris C., Gunn S. On a class of a support vector kernels based on frames in function Hilbert spaces, Neural Comput. 13 (9), 1975-1994 (2001).

[18] Rakotomamonjy A., Canu S. Frames, reproducing kernels, regularization and learning, Journal of Machine Learning Research 6, 1485-1515 (2005).

[19] Халмош П. Гильбертово пространство в задачах (Мир, М., 1970). 
[20] Marcus A.W., Spielman D.A., Srivastava N. Interlacing families II: mixed characteristic polynomials and the Kadison-Singer problem, Ann. Math. 182 (1), 327-350 (2015).

[21] Ахиезер Н.И., Глазман И.М. Теория линейных операторов в гилъбертовом пространстве (Наука, М., 1966).

[22] Totik V. Recovery of $H^{p}$-functions, Proc. Amer. Math. Soc. 90 (4), 531-537 (1984).

[23] Терехин П.А. Системы представления и проекиии базисов, Матем. заметки 75 (6), $944-947$ (2004).

[24] Терехин П.А. Банаховы фреймы в задаче аффинного синтеза, Матем. сб. 200 (9), 127-146 (2009).

[25] Терехин П.А. Фреймы в банаховом пространстве, Функц. анализ и его прилож. 44 (3), 50-62 (2010).

[26] Schaefer H.H., Wolff M.P. Topological vector spaces (2nd ed., Grad. Text in Math., Springer-Verlag, New York, 1999).

Константин Сергеевич Сперанский

Саратовский национальный исследовательский

государственный университет им. Н.Г. Чернышевского,

ул. Астраханская, д. 83, г. Саратов, 410012, Россия,

e-mail: konstantin.speransky@yahoo.com

Павел Александрович Терехин

Саратовский национальный исследовательский

государственный университет им. Н.Г. Чернышевского,

ул. Астраханская, д. 83, г. Саратов, 410012, Россия,

e-mail: terekhinpa@mail.ru

\section{K.S. Speransky and P.A. Terekhin}

\section{On existence of frames based on the Szegö kernel in the Hardy space}

Abstract. It is well-known that a sequence of functions constructed by sampling of the Szegö kernel cannot be the Duffin-Shaeffer frame for the Hardy space on the unit disk. In this paper we show that by using the more general concept of a frame the problem of existence of a frame based on the Szegö kernel has a solution.

Keywords: Duffin-Schaeffer frames, Banach frames, framing model, reproducing kernel Hilbert space, Hardy space, Szegö kernel.

Konstantin Sergeevich Speransky

Saratov State University,

83 Astrakhanskaya str., Saratov, 410012 Russia,

e-mail: konstantin.speransky@yahoo.com

Pavel Aleksandrovich Terekhin

Saratov State University,

83 Astrakhanskaya str., Saratov, 410012 Russia,

e-mail: terekhinpa@mail.ru 